\title{
Heat dissipative solitons in optical fibers
}

\author{
N. Akhmediev ${ }^{\text {a }}$, P.St.J. Russell ${ }^{\text {b }}$, M. Taki ${ }^{\text {c }}$, J.M. Soto-Crespo ${ }^{\text {d,* }}$ \\ a Optical Sciences Group, Research School of Physical Sciences and Engineering, The Australian National University, Canberra ACT 0200, Australia \\ ${ }^{\mathrm{b}}$ Max-Planck Research Group (IOIP), University Erlangen-Nuremberg, Erlangen, Germany \\ ${ }^{\mathrm{c}}$ Laboratoire de Physique des Lasers, Atomes et Molécules, UMR CNRS 8523, Centre d'Études et de Recherches Lasers et Applications, \\ Université des Sciences et Technologies de Lille, 59655 Villeneuve d'Ascq cedex, France \\ d Instituto de Óptica, C.S.I.C., Serrano 121, 28006 Madrid, Spain \\ Received 18 September 2007; accepted 22 September 2007 \\ Available online 2 October 2007 \\ Communicated by V.M. Agranovich
}

\begin{abstract}
We propose a one-dimensional model governing the propagation of heat waves in an optical fiber (the "fiber fuse"). The model has solutions in the form of high temperature localized waves moving towards the input end of the fiber, fueled by the laser power. These waves can be ignited by local heating at any point along the fiber. The effect of such a wave is irreversible damage to the fiber core. The phenomenon was observed earlier by Hand and Russell, when locally heating a fiber through which CW light of modest intensity was propagating. This induced self-destruction of the optical fiber core.
\end{abstract}

(C) 2007 Elsevier B.V. All rights reserved.

PACS: 42.65.-k; 47.20.Ky; 47.25.Qv

\section{Introduction}

Hand and Russell observed the fascinating phenomenon of a glowing thermal shock wave traveling along an optical fiber pumped by laser light of modest total power [1]. The striking appearance of the phenomenon caused it to be named the "fiber fuse". Thermally induced catastrophic breakdown of the glass material in the fiber is the main mechanism for the glowing spot moving in the opposite direction to the pump light. Similar observations were made by Kashyap and Blow [2]. The phenomenon has been observed in both single mode and multimode fibers. It is an example of a one-dimensional dissipative soliton that exists solely as a result of an external energy pump. It can be studied using a phenomenological approach. In this work, we propose a one-dimensional model that describes the basic features of the effect.

The usual way of describing optical effects in a fiber is a one-dimensional representation with all magnitudes depending

\footnotetext{
* Corresponding author.

E-mail address: iodsc09@io.cfmac.csic.es (J.M. Soto-Crespo).
}

on the longitudinal coordinate $x$ along the fiber and time $t$. The main process taking place in the fiber fuse is the local heating of the fiber induced by the absorption of the optical field that has a certain fixed modal transverse profile. Thus, the heated part of the optical fiber can be described by the heat transfer equation with the source term, i.e.,

$T_{t}=D \frac{\partial^{2} T}{\partial x^{2}}+\chi \alpha I-k\left(T-T_{0}\right)$,

where $T(x, t)$ is the local temperature of the fiber averaged over its cross section, $D$ is the diffusion coefficient, $\alpha(x, t)$ is the absorption coefficient, also averaged over the fiber cross section and $I(x, t)$ is the light power inside the fiber, integrated over the fiber cross section. All the functions $T, I$ and $\alpha$ are positive real ones which, in our approximation, depend on two variables $(x, t)$. The product $\alpha I$ in Eq. (1) represents the rate of energy lost by the optical field in the fiber. It serves as the heat source in the problem. The coefficient $\chi$ takes into account geometrical factors and the loss of energy in the transverse direction; in our approximation it can be considered as a constant. The last term $k\left(T-T_{0}\right)$ represents the relaxation of the temperature to 
ambient temperature $T_{0}$ after the heating process moves away from a particular position.

Irreversible optical damage to the material of fiber can be described by another equation. For short pieces of fiber (tens of meters in the experiment) before heating, the absorption can be completely ignored $(\alpha=0)$. When the temperature in the fiber approaches $1200^{\circ} \mathrm{C}$, the guiding properties of the fiber deteriorate. Thus, above a critical temperature $T_{1}$, the absorption coefficient grows with increasing local temperature. The fiber remains damaged after that. Hence, the absorption coefficient afterwards remains at the level reached during the heating cycle. According to this model, the rate of change of the absorption coefficient must be a function of the temperature, $T(x, t)$. Following experimental data [3], we approximate it with a linear function that starts at the threshold temperature, namely:

$\alpha_{t}=F(T)= \begin{cases}0, & \text { if } T<T_{1}, \\ m\left(T-T_{1}\right), & \text { if } T>T_{1},\end{cases}$

where $m$ is a factor $\left(\approx 10^{-3} \mathrm{~m}^{-1} \mathrm{~s}^{-1} \mathrm{~K}^{-1}\right)$ that depends on the specific mechanism of glass damage and the transverse dimensions of the fiber. The critical temperature $T_{1}$ at which the damage starts is known to be around $1200^{\circ} \mathrm{C}$. A smoothly varying function rather than (2) could be used in a more detailed description, but the linear part of this function clearly defines the main features of the effect.

The laser power propagating along the fiber is absorbed when the temperature goes above the critical one, and the absorption starts to differ from zero, as Eq. (2) describes. Therefore, the power must follow the equation:

$I_{x}=-\alpha I$.

The set of these three equations gives a simple one-dimensional description of the phenomenon. In a mathematical sense, it is a complete model that serves to state the problem on a phenomenological basis.

Let us introduce the variable $\tau=m\left(T-T_{0}\right)$ which is the normalized excess of $T$ above ambient temperature $T_{0}$. Then the following set of equations describes the process:

$\tau_{t}=D \tau_{x x}+m \chi \alpha I-k \tau$,

$\alpha_{t}=F(\tau)$,

$I_{x}=-\alpha I$.

All dependent variables here are positive. The function $F(\tau)$ is a step function:

$F(\tau)= \begin{cases}0, & \text { if } \tau<\tau_{1}, \\ \left(\tau-\tau_{1}\right), & \text { if } \tau>\tau_{1},\end{cases}$

where $\tau_{1}=m\left(T_{1}-T_{0}\right)$.

The last equation in (4) can be written in the form: $(\log I)_{x}=$ $-\alpha$ which has the following solution

$I(x, t)=I_{0} \exp \left[-\int_{-\infty}^{x} \alpha(y, t) d y\right]$

where $I_{0}$ is the initial light power in the fiber. When $\alpha=0$, the laser power along the fiber is constant $I(x, t)=I_{0}$. The power decreases in areas where the absorption becomes nonzero. Substituting this equation into the dynamical system, we obtain a set of integro-differential equations relative to $\tau$ and $\alpha$ :

$\tau_{t}=D \tau_{x x}+J_{0} \alpha \exp \left[-\int_{-\infty}^{x} \alpha(y, t) d y\right]-k \tau$,

$\alpha_{t}=F(\tau)$.

Here $J_{0}=m \chi I_{0}$ is the normalized optical power at the input end of the fiber $x=0$. The set of equations (7) is similar to the problems of front propagation in biology [4] except for the integral and the function $F(\tau)$, which has a discontinuous derivative. The integral in Eq. (7) describes nonlocal effects in the problem.

Suppose now, that we are solving this set of equations over the interval $0<x<L$ where $L$ is the length of the fiber. We are interested in the evolution of the variables $\tau$ and $\alpha$ at $t>0$. Under these conditions, the set of equations (7) must be supplemented by the initial conditions. If the temperature of the fiber at $t=0$ is below the critical value $\tau_{1}$, it will return to ambient temperature with a relaxation rate $k$ provided that $\alpha(x, t=0)$ is also zero. The process of heat wave propagation needs to be ignited at a certain point along the fiber by local heating above the threshold temperature. The wave cannot self-start without this initial condition. The initial conditions that start the process can be described by the equations:

$\alpha(x, 0)=0$,

$\tau(x, 0)=\left(\tau_{1}+\Delta\right) \exp \left[-q\left(x-x_{0}\right)^{2}\right]$,

where any small $\Delta$ provides excess temperature above the threshold at point $x_{0}$. This results in a nonzero function $F(\tau)$ that ignites the heat-wave. The value $q$ defines the size of the fiber region being initially heated. It does not influence the form of the traveling wave that moves away from the point $x=x_{0}$ afterwards. On the other hand, the boundary condition for $I$ at $x=0$ is given by:

$I(0, t)=I_{0}$.

Eq. (9) gives the pump power of laser light coupled into the fiber at the input. The formulae (7), (8) and (9) constitute a complete set of equations that defines mathematically the problem.

Numerical simulations of the above equations have been carried out with a split step Fourier method. An example of a traveling wave solution obtained numerically is shown in Figs. 1, 2 and 3 for the variables $\tau, I$ and $\alpha$ respectively. The initial heat in this example was applied at the point $x=50$. In this numerical example, the temperature increases and the front moves to the input end of the fiber at constant speed as expected from the experiment [1]. Similar behavior to that observed in the above figures are obtained for other values of the parameters. However, the soliton does not move for every set of parameters. The heat dissipation can be fast enough to prevent destruction of the fiber at points other than the initial region around $x=x_{0}$.

In order to describe the wave front analytically, we look for traveling wave solutions to the set of equations (7). Introducing the new variable $\xi=x+v t$, where $v$ is the traveling wave 


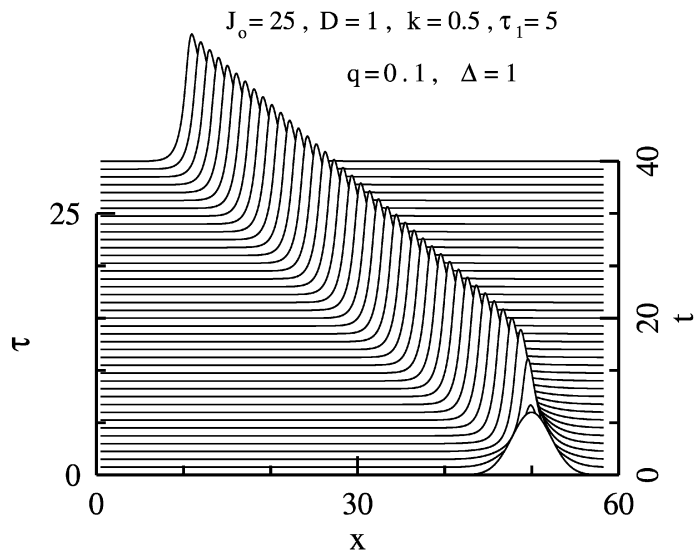

Fig. 1. Evolution of the normalized fiber temperature $\tau$ with time $t$. Parameters of the simulation are: $D=1, \tau_{1}=5, J_{0}=25.0, k=0.5$. The input is given by Eq. (8) with $q=0.1$, and $\Delta=1$.

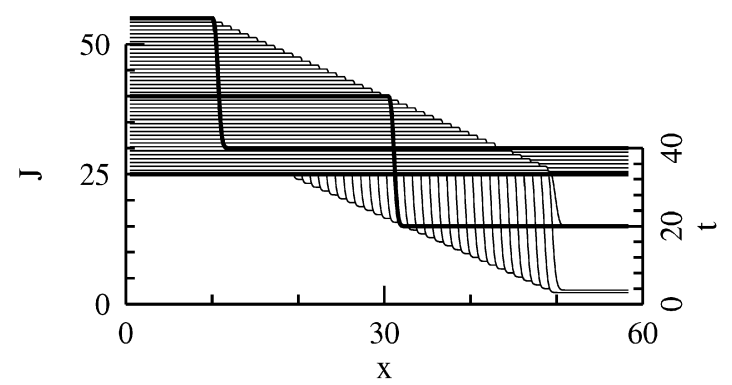

Fig. 2. Evolution of the normalized laser power $J(x, t)=m \chi I(x, t)$ with time $t$. The intensities at $t=0,20$ and 40 are marked with a thicker line for clarity.

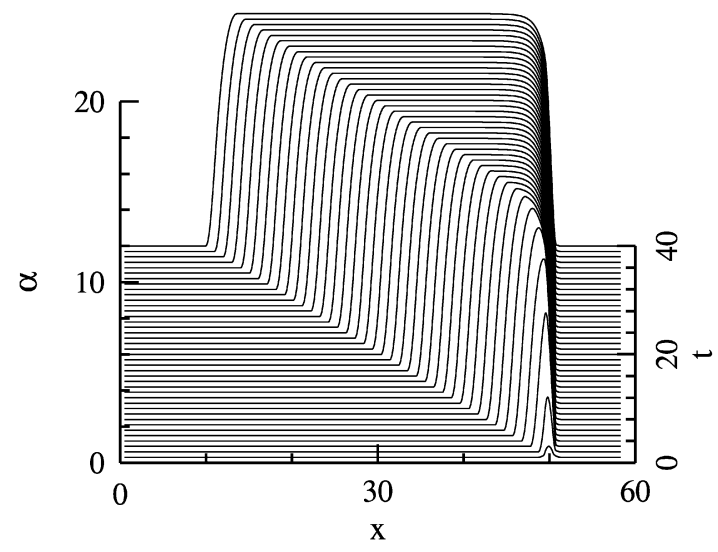

Fig. 3. Evolution of the absorption coefficient $\alpha$ with time.

velocity, we obtain

$v \tau_{\xi}=D \tau_{\xi \xi}+J_{0} \alpha \exp \left(-\int_{-\infty}^{\xi} \alpha d x\right)-k \tau$,

$v \alpha_{\xi}=F(\tau)$.

This is a set of ordinary integro-differential equations where $\alpha(\xi)$ and $\tau(\xi)$ depend only on one variable $\xi$. As numerical simulations show, the front has a fixed shape after the initial condition converges to it. Let us suppose that the temperature is equal to $\tau_{1}$ at $\xi=0$. Then $\alpha=0$ when $\xi<0$. Thus, the set of equations (10) at $\xi<0$ takes the form:

$v \tau_{\xi}=D \tau_{\xi \xi}-k \tau$,

$\alpha=0$.

The first of these equations is a second order linear equation with constant coefficients. Its solution can be easily found to be:

$\tau(\xi)=A e^{\mu_{1} \xi}+B e^{\mu_{2} \xi}$,

where $\mu_{1,2}$ are the roots of a second order polynomial that are given by

$\mu_{1,2}=\frac{v}{2 D} \pm \sqrt{\frac{v^{2}}{4 D^{2}}+\frac{k}{D}}$.

Clearly, $\mu_{1}>0$ and $\mu_{2}<0$, as $k / D$ is positive. Hence, for the solution to go to zero at the left-hand side, $B$ has to be zero. To satisfy the boundary condition at $\xi=0$, we take $A=\tau_{1}$. The derivative of $\tau(\xi)$ at $\xi=0$ has to be matched with the derivative of $\tau(\xi)$ at $\xi>0$. We have $\tau=\tau_{1} e^{\mu_{1} \xi}$ and the derivative $\tau^{\prime}(0)=$ $\tau_{1} \mu_{1}$.

At $\xi>0$, we have to solve the equations:

$v \tau_{\xi}=D \tau_{\xi \xi}+J_{0} \alpha \exp \left(-\int_{0}^{\xi} \alpha d x\right)-k \tau$,

$v \alpha_{\xi}=\left(\tau-\tau_{1}\right)$

These have to be supplemented with the boundary conditions:

$\alpha(0)=0, \quad \tau(0)=\tau_{1}, \quad \tau^{\prime}(0)=\tau_{1} \mu_{1}$,

$\alpha(\infty)=$ const.

To solve the set of equations (13), we can reduce them to a single differential equation. The second equation in (13) provides the expression for $\tau$ :

$\tau=\tau_{1}+v \alpha \xi$.

It can be differentiated twice to give: $\tau_{\xi}=v \alpha_{\xi \xi}$ and $\tau_{\xi \xi}=$ $v \alpha_{\xi \xi \xi}$. Substituting these derivatives into the first equation (13) gives:

$D \alpha_{\xi \xi \xi}-v \alpha_{\xi \xi}-k \alpha_{\xi}+\frac{J_{0}}{v} \alpha \exp \left(-\int_{0}^{\xi} \alpha d x\right)=\frac{k}{v} \tau_{1}$.

The boundary conditions now are:

$\alpha(0)=0, \quad \alpha^{\prime}(0)=0, \quad \alpha^{\prime \prime}(0)=\tau_{1} \mu_{1} / v$,

$\alpha(\infty)=$ const

The last equation can also be written in the form:

$D \alpha_{\xi \xi \xi}-v \alpha_{\xi \xi}-k \alpha_{\xi}+\frac{J_{0}}{v}\left[\exp \left(-\int_{0}^{\xi} \alpha d x\right)\right]_{\xi}=\frac{k}{v} \tau_{1}$. 


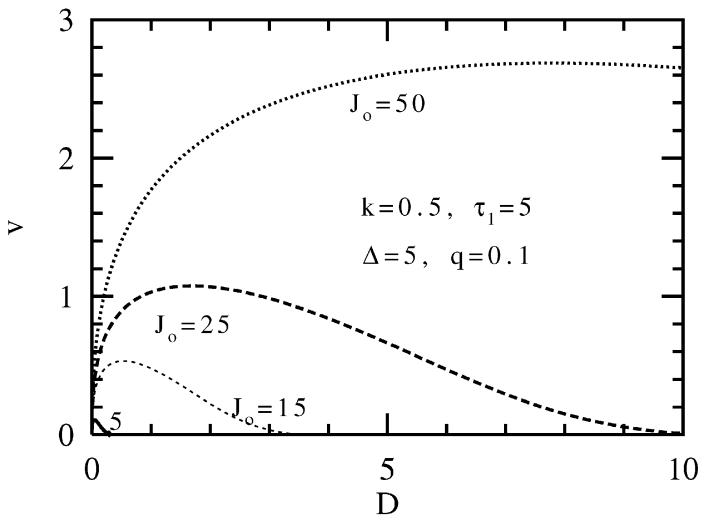

Fig. 4. Velocity of the front wave vs. $D$ for four values of $J_{0}$.

Integrating this equation once results in:

$D \alpha_{\xi \xi}-v \alpha_{\xi}-k \alpha+\frac{J_{0}}{v} \exp \left(-\int_{0}^{\xi} \alpha d x\right)=\frac{k}{v} \tau_{1} \xi+C$,

where $C$ is a constant of integration. Now, we define $\phi(\xi)=$ $\int_{0}^{\xi} \alpha d x$ which means: $\phi_{\xi}=\alpha$. Thus, Eq. (16) can be written in the form:

$D \phi_{\xi \xi \xi}-v \phi_{\xi \xi}-k \phi_{\xi}+\frac{J_{0}}{v} e^{-\phi}=\frac{k}{v} \tau_{1} \xi+C$.

This is a nonlinear, non-autonomous differential equation of third order. To find how the velocity depends on the parameters of the problem, it has to be solved together with the boundary conditions: $\phi_{\xi}(0)=0, \phi_{\xi \xi}(0)=\tau_{1}, \phi_{\xi \xi \xi}(0)=\tau_{1} \mu_{1}$ and $\phi_{\xi}(\infty)=$ const. The equations still contain two unknown constants which can be found from the limiting cases. Solutions of (17) will be presented elsewhere. In what follows below, we find the velocity $v$ using direct numerical simulations of equations (4) as described above. An advantage is that, this way, we find only robust front solutions avoiding complications related to stability issues.

Generally, the velocity of the moving front depends on four parameters $J_{0}, D, \tau_{1}$ and $k$ and can take values in a large range starting from zero. To give an example, the dependence of the velocity on the diffusion parameter $D$ for several input powers $J_{0}$ is shown in Fig. 4. The velocity is zero at zero $D$ because there is no heat transfer in this case. It increases as the square root of $D$ at small $D$ but decreases back to zero at large $D$. The reason for this decrease is fast heat transfer along the fiber which reduces the rate of fiber destruction.

Fig. 5 shows a plot of $V$ vs. normalized input power $J_{0}$. When the input power $J_{0}$ is small, the source of heat keeps the temperature below the critical value. The damaged region of the

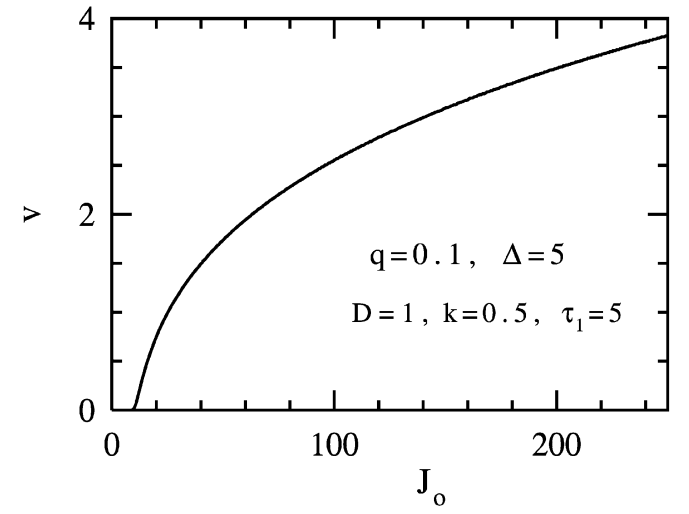

Fig. 5. Velocity of the front wave vs. the normalized input power $J_{0}$.

fiber does not move in this case. Only when the laser light creates sufficient heat to raise the temperature above the threshold, does the front start to move along the fiber. Above this threshold, the dependence of the velocity $v$ on $J_{0}$ can roughly be described by a square root function. If the parameters of the problem are given in physical units: $D\left[\frac{\mathrm{m}^{2}}{\mathrm{Ks}}\right], \alpha\left[\mathrm{m}^{-1}\right], k\left[\mathrm{~s}^{-1}\right]$, then the vertical scale in Figs. 4 and 5 is of the order of $\left[\mathrm{m} \mathrm{s}^{-1}\right]$. Comparing the present Fig. 5 with Fig. 3 of Ref. [3] shows that our simple model gives qualitatively good description of the phenomenon.

In conclusion, we have proposed a one-dimensional model governing the propagation of the fiber fuse along an optical fiber as observed earlier experimentally by Hand and Russell. The solution of this model in the form of traveling front wave can be considered as another example of a dissipative soliton [5]. This soliton is a high temperature localized wave moving towards the input end of the fiber from the point of ignition causing irreversible damage to the fiber core. It is fueled by modest laser powers and heat is dissipated in the space around the fiber after the wave is passed.

\section{Acknowledgements}

N.A. acknowledges support from the Australian Research Council and University of Lille. The work of J.M.S.C. was supported by the M.E.y C. under contract FIS2006-03376.

\section{References}

[1] D.P. Hand, P.St.J. Russell, Opt. Lett. 13 (1988) 767.

[2] R. Kashyap, K.J. Blow, Electron. Lett. 24 (1988) 47.

[3] D.P. Hand, J.E. Townsend, P.St.J. Russell, Optical damage in fibers: The fiber fuse, Digest of CLEO, Anaheim (OSA), 1988.

[4] J.D. Murray, Mathematical Biology, Springer, Berlin, 1993 (Chapter 13).

[5] N. Akhmediev, A. Ankiewicz (Eds.), Dissipative Solitons, Springer, Heidelberg, 2005. 